PROCEEDINGS OF THE

AMERICAN MATHEMATICAL SOCIETY

Volume 42, Number 1, January 1974

\title{
AN EXAMPLE OF A LOCAL FLOW ON A MANIFOLD ${ }^{1}$ DENIS L. BLACKMORE
}

\begin{abstract}
Let $p$ be a point of a smooth $n$-dimensional manifold. If $n$ is even it is easy to construct a local flow about $p$ such that $p$ is an isolated critical point and no orbit except the stationary one at $p$ has $p$ as a limit point. We call such a flow a nonnull flow about $p$ (NN-flow). Mendelson has conjectured that NN-flows do not exist on odd dimensional manifolds. We show that Mendelson's conjecture is false by constructing an NN-flow on any smooth manifold whose dimension is an odd integer exceeding one.
\end{abstract}

1. Introduction. Let $p$ be a point of a finite-dimensional smooth manifold $M$ (here smooth means of class $C^{\infty}$ ) with tangent bundle $T(M)$, and let $(U, h)$ be a coordinate system about $p$. A continuous vector field $X: U \rightarrow T(M)$ generates a local flow in a neighborhood of $p$, provided that the vector field has unique integral curves (see [1], [2], [3], and [4] for details).

If the dimension of $M(\operatorname{dim} M)$ is even, it is easy to construct a local flow about $p$ such that $p$ is an isolated critical point and no orbit except the stationary one at $p$ has $p$ as a limit point; we shall call such a flow an NN-flow about $p$ (NN denotes the fact that there are no null solutions based at $p$ ). In contrast to the even dimensional case, the question of whether or not there exists an NN-flow about any point of $M$ when $\operatorname{dim} M$ is an odd integer exceeding one (clearly if $\operatorname{dim} M=1$, there exists no $\mathrm{NN}$-flow) is a fairly deep one and, to my knowledge, has not been resolved in the literature. The purpose of this paper is to answer this question; in particular, we demonstrate the falsehood of a conjecture of Mendelson that there exists no NN-flow when $\operatorname{dim} M$ is odd. We accomplish this by obtaining an example which provides a proof of the following:

THEOREM 1. If $\operatorname{dim} M$ is an odd integer greater than one, then there exists a vector field in a neighborhood of any point $p$ of $M$ which generates an $N N$-flow about $p$.

The proof of Theorem 1 consists of constructing a local flow about $p$ with the property that every orbit other than the stationary one at $p$ is separated from $p$ by an invariant torus.

Presented to the Society, November 25, 1972; received by the editors February 5 , 1973.

AMS (MOS) subject classifications (1970). Primary 34C40, 34C35; Secondary 54H20, 58F99.

1 This research was supported in part by a NCE Research Initiation Grant.

(c) American Mathematical Society 1974 
I wish to thank Professor P. Mendelson for bringing his conjecture to my attention. The discussions we had concerning this problem, and especially the doubts he expressed about the truth of his conjecture, were instrumental in the writing of this paper.

2. Proof of Theorem 1 by example. Let $R^{n}$ denote euclidean $n$-space with element $x=\left(x_{1}, \cdots, x_{n}\right)$, and let 0 denote the origin in $R^{n}$. We first note that it suffices to construct a vector field about the origin in $R^{3}$ which generates an NN-flow about 0 . Indeed let $M$ be a smooth manifold with $\operatorname{dim} M=2 n+1(n \geqq 1)$, and let $p$ be any point of $M$. We may assume that the coordinate system $(U, h)$ about $p$ is such that $h(U)=R^{2 n+1}$, and $h(p)=0$. Suppose that $W$ is an open neighborhood of 0 in $R^{3}$ and $F: W \rightarrow R^{3}$ is a vector field which generates an NN-flow about 0 . Recall that the vector field $G:\left(x_{1}, x_{2}\right) \rightarrow\left(-x_{2}, x_{1}\right)$ is the standard center at the origin in $R^{2}$; in particular it generates an NN-flow about the origin on the whole plane. Taking the cartesian product of $F$ and the $(n-1)$-fold cartesian product of $G$, namely $H=F \times \prod_{m=1}^{n-1} G$, we obtain a vector field $H: W \times$ $R^{2(n-1)} \rightarrow R^{2 n+1}$ which generates an NN-flow about the origin in $R^{2 n+1}$. Let the restriction $h \mid h^{-1}(W)$ be denoted by $g$. Then $g^{*}(H)$, where $g^{*}$ is the pull-back as in Sternberg [4, p. 89], generates an NN-flow about $p$ on $M$.

We now proceed with the construction of an example in $R^{3}$. The process will be completed in a series of simple steps. The idea is to inductively define smooth, nonvanishing vector fields in a sequence of nonoverlapping spherical shells of decreasing outer radius and thickness. Each of the shells is centered at the origin, and their union is the solid ball in $R^{3}$ except for the origin; the field is then defined to be zero at the origin. The vector field in each shell is constructed in virtually the same way so as to have the additional property that the shell contains a torus which is invariant with respect to the flow generated.

First we define a nonvanishing vector field on a typical shell which extends a prescribed vector field on its boundary. We shall need the following definitions: Let $\|\cdot\|$ denote the euclidean norm on $R^{n}$. If $N$ is a manifold with boundary, we denote its interior by Int $N$ and its boundary by $\partial N$. Define the sets

$$
\begin{aligned}
& B(r)=\left\{x \in R^{3}:\|x\| \leqq r(0<r)\right\}, \quad S(r)=\partial B(r), \\
& Q(r)=\left\{x \in R^{3}: r \leqq\|x\| \leqq 8 r(0<r)\right\} .
\end{aligned}
$$

We shall begin with the shell $Q(r)$, where $r$ is any positive number. The vector field on $\partial Q(r)$ is prescribed as follows:

$$
\begin{aligned}
V_{0}(x) & =(0, r, 0), & & x \in S(r), \\
& =(0,0,8 r), & & x \in S(8 r) .
\end{aligned}
$$


This is extended to a nonvanishing vector field $V_{1}(x)$ on $Q(r)$ by defining

$$
V_{1}(x)=(7 r)^{-1}(0,(8 r-\|x\|) r,(\|x\|-r) 8 r) \quad(x \in Q(r)) .
$$

This field is smooth (in fact analytic) on $Q(r)$. We note that

$$
\left\|V_{1}\right\|=\max \left\{\left\|V_{1}(x)\right\|: x \in Q(r)\right\} \leqq r \sqrt{ } 65 .
$$

Next, we modify the vector field $V_{1}$ by imbedding an invariant torus in the shell. In what follows let the disk of radius $r$ in $R^{2}$ be designated by $D(r)=\left\{x \in R^{2}:\|x\| \leqq r\right\}$, and let $d\left(A, A^{\prime}\right)$ denote the distance between two subsets $A$ and $A^{\prime}$ of $R^{3}$.

Let $\phi(t)$ be a nondecreasing, smooth, real-valued function on $R$ such that

$$
\begin{gathered}
\phi(t)=0 \text { for } t \leqq \pi / 6, \quad \phi(t)=\pi \quad \text { for }(5 \pi / 6) \leqq t \leqq(7 \pi / 6), \\
\phi(t)=2 \pi \quad \text { for } t \geqq(11 \pi / 6) .
\end{gathered}
$$

Then

$$
C=\left\{x \in R^{3}: x=x(t)=(-2 r+2 r \cos \phi(t), 2 r \sin t, 0), 0 \leqq t \leqq 2 \pi\right\}
$$

is a smooth, simple, closed curve in the $x_{1} x_{2}$-plane which contains the portion of the $x_{1}$-axis where $-r \leqq x_{2} \leqq r$. The set

$$
T=\left\{x \in R^{3}: d(x, C) \leqq r\right\}
$$

is a smooth submanifold of $R^{3}$ with boundary

$$
\partial T=\left\{x \in R^{3}: d(x, C)=r\right\} .
$$

We readily verify the following properties:

(i) $T$ and $\partial T$ are, respectively, a solid torus and a torus; that is, they are respectively diffeomorphic to $\partial D(1) \times D(1)$ and $\partial D(1) \times \partial D(1)$.

(ii) $T$ contains the cylinder $\left\{x \in R^{3}: x_{1}^{2}+x_{3}^{2}=r^{2},-r \leqq x_{2} \leqq r\right\}$.

(iii) Let $T^{\prime}=T-$ Int $B(r)$. Then $T^{\prime} \subset Q(r)$.

(iv) Let $T_{\varepsilon}=\left\{x \in R^{3}: d(x, T) \leqq \varepsilon\right\}$. If $0<\varepsilon<r$, then $T_{\varepsilon}$ and $\partial T_{\varepsilon}$ are respectively a solid torus and a torus. Also $\left(T_{\varepsilon}-\operatorname{Int} B(r)\right) \subset Q(r)$, and $d\left(T_{\varepsilon}, S(8 r)\right)>r$. The solid torus $T$ has a parametrization given by

$$
x_{1}=-2 r+2 r \cos \phi(u)\left[1+\left(\sin ^{2} u+\cos ^{2} \phi(u)\right)^{-1 / 2} w \cos v\right],
$$

$$
\begin{aligned}
& x_{2}=2 r \sin u\left[1+\left(\sin ^{2} u+\cos ^{2} \phi(u)\right)^{-1 / 2} w \cos v\right], \\
& x_{3}=w \sin v,
\end{aligned}
$$

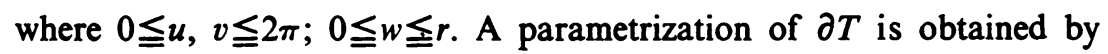
setting $w=r$ in (2).

Fixing $v$ and $w$ in (2) and allowing $u$ to vary, we obtain a smooth, simple, closed curve in a plane which is parallel to the $x_{1} x_{2}$ plane. Each of these 
curves has counterclockwise orientation; the curves will be called generators of $T$. We note that $T$ is partitioned by its generators. We use these generators to define a vector field $V_{2}$ on $T^{\prime}$. Set $V_{2}(x)$ equal to the vector obtained by taking the velocity vector at $x$ of the generator passing through this point and normalizing it to a magnitude of $r$. In this way we obtain a smooth vector field $V_{2}$ on $T^{\prime}$ such that $\left\|V_{2}(x)\right\|=r$ and $V_{2}(x)$ lies on the $x_{1} x_{2}$-plane for each $x$ in $T^{\prime}$. In view of (1), the definition of $C$, and property (ii), we see also that $V_{2}(x)$ is a smooth extension of the restriction $V_{1} \mid S(r)$.

At present, let $\varepsilon$ be subject only to the restriction $0<\varepsilon<r$; later we shall further restrict it. We define a vector field $V_{3}$ on $\left(Q(r)-\operatorname{Int} T_{\varepsilon}\right) \cup T^{\prime}$ by

$$
\begin{aligned}
V_{3}(x) & =V_{1}(x), & & x \in\left(Q(r)-\text { Int } T_{\varepsilon}\right), \\
& =V_{2}(x), & & x \in T^{\prime} .
\end{aligned}
$$

Now we extend this to a vector field $V_{5}$ on $Q(r)$ by the following "normal extension" process: At a point $x^{\prime} \in \partial T$ extend an outward normal vector until it intersects $\partial T_{\varepsilon}$ at the point $x^{\prime \prime}$. The resulting line segment $\left[x^{\prime}, x^{\prime \prime}\right]$, which is of length $\varepsilon$, may be parametrized $x=x(t), 0 \leqq t \leqq \varepsilon$, where $x(0)=$ $x^{\prime}$, and $x(\varepsilon)=x^{\prime \prime}$. A vector field is defined on the boundary of the segment: $V_{2}\left(x^{\prime}\right)$ at $x^{\prime}$ and $V_{1}\left(x^{\prime \prime}\right)$ at $x^{\prime \prime}$. Let $\psi(t)$ be a smooth, nondecreasing, realvalued function on $R$ such that $\psi(t)=0$ for $t \leqq \varepsilon / 3$, and $\psi(t)=1$ for $t \geqq 2 \varepsilon / 3$. Define $V_{4}$ on $\left[x^{\prime}, x^{\prime \prime}\right]$ by

$$
V_{4}(x(t))=(1-\psi(t)) V_{2}\left(x^{\prime}\right)+\psi(t) V_{1}\left(x^{\prime \prime}\right) \quad(0 \leqq t \leqq \varepsilon) .
$$

Repeating this process at each point of $\partial T$, we obtain a vector field $V_{4}$ defined on $T_{\varepsilon}$-Int $T$ which is a smooth extension of the given vector field on the boundary of this set. Define

$$
\begin{aligned}
V_{5}(x) & =V_{3}(x), & & x \in\left(Q(r)-\text { Int } T_{\varepsilon}\right) \cup T^{\prime}, \\
& =V_{4}(x), & & x \in\left(T_{\varepsilon}-\text { Int } T\right) .
\end{aligned}
$$

This field is smooth on $Q(r)$, and tangent to each $\partial T_{\delta}(0 \leqq \delta \leqq \varepsilon / 3)$. Moreover it is nonvanishing. Indeed, since $V_{3}(x)$ is never zero, the only way a zero can arise is in $V_{4}(x)$. But this can occur only if at some stage of the normal extension the vectors $V_{2}\left(x^{\prime}\right)$ and $V_{1}\left(x^{\prime \prime}\right)$ oppose one another. In view of (1) and the definition of $V_{2}$, this is impossible.

A final modification makes the field constant in a neighborhood of $S(8 r)$. We accomplish this by redefining the field in the shell $Q_{\varepsilon}=B(8 r)-$ Int $B(8 r-\varepsilon)$ so that it agrees with $V_{5}$ on $\partial Q_{\varepsilon}$. Observe that property (iv) together with the restriction $0<\varepsilon<r$ guarantees that any such alteration does not change the field on $T_{\varepsilon}-\operatorname{Int} B(r)$. In view of the continuity of $V_{5}$ and the compactness of $S(8 r)$, we can, and do, select $\varepsilon$ so small that the vectors defined at the endpoints of the segment of every ray through 0 
joining $S(8 r-\varepsilon)$ and $S(8 r)$ are not in opposition. Redefining the field in $Q_{\varepsilon}$ by the process of normal extension described above, we obtain a smooth, nonvanishing vector field $V_{6}$ which is an extension of $V_{5} \mid \partial Q_{\varepsilon}$. Define

$$
\begin{aligned}
F(x) & =V_{5}(x), & & x \in\left(Q(r)-Q_{\varepsilon}\right), \\
& =V_{6}(x), & & x \in Q_{\varepsilon} .
\end{aligned}
$$

Then $F$ is a smooth, nonvanishing vector field on $Q(r)$. It is tangent to $\partial T$, and therefore generates a flow on $Q(r)$ for which $\partial T$ is an invariant surface. By retracing the modifications, we compute that

$$
\|F\| \leqq r \sqrt{ } 130 .
$$

It is now easy to construct the desired example. Let $Q=Q(1)$, let $F$ be the vector field on $Q$ described above, and let $T$ be the solid torus associated with the field. Let the map $f_{1}: R^{3} \rightarrow R^{3}$ be the contraction $f_{1}(x)=x / 8$, and let $f_{2}: R^{3} \rightarrow R^{3}$ be a clockwise rotation of 45 degrees about the $x_{1}$-axis. Define $f$ to be the composite $f=f_{1} \circ f_{2}$. Clearly $f(Q(r))=$ $Q(r / 8)$. Let $f_{*}$ be the map of tangent bundles induced by $f$ (i.e. the

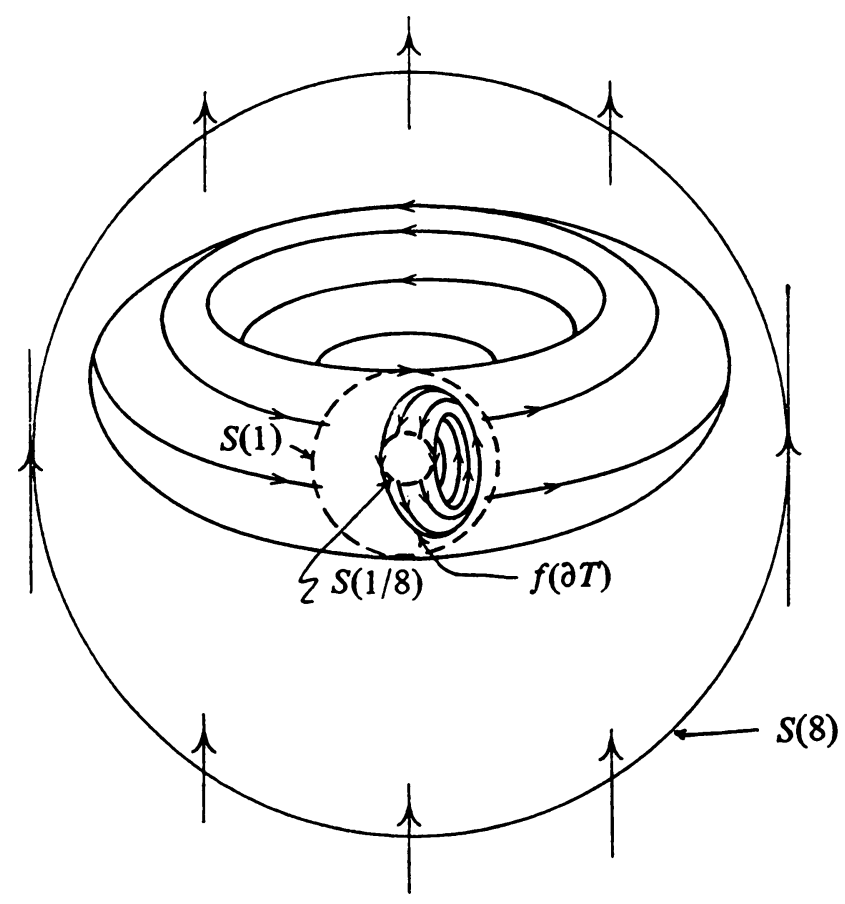

FIGURE 1 
differential of $f$ ), and let $f^{n}$ and $f_{*}^{n}$ be the $n$-fold composite of $f$ and $f_{*}$, respectively. As usual, we shall denote the vector field $f_{*}^{n} \circ F \circ f^{-n}$ on $f^{n}(Q)$ by $f_{*}^{n}(F)$. We note that $f_{*}^{n}(F)$ is nonvanishing for every nonnegative integer $n$. The following observations are the basis for the example:

(4a) For each $n=0,1,2, \cdots, f^{n}(\partial T)$ is a torus contained in the shell $f^{n}(Q)$, and the vector field $f_{*}^{n}(F)$ is tangent to the surface $f^{n}(\partial T)$.

(4b) The union $B(8)-\{0\}=\bigcup_{n=0}^{\infty} f^{n}(Q)$ is nonoverlapping, and for each nonnegative integer $n$, the fields $f_{*}^{n+1}(F)$ and $f_{*}^{n}(F)$ defined on $f^{n+1}(Q)$ and $f^{n}(Q)$, respectively, agree on the intersection of their domains. Moreover, the unique extension of $f_{*}^{n+1}(F)$ and $f_{*}^{n}(F)$ to $f^{n+1}(Q) \cup f^{n}(Q)$ is smooth.

The desired vector field on $B(8)$ (see Figure 1) is defined by

$$
\begin{aligned}
X(x) & =f_{*}^{n}(F)(x), & & x \in f^{n}(Q), n=0,1,2, \cdots, \\
& =0, & & x=0 .
\end{aligned}
$$

By virtue of (4b) and (3) which implies that $\left\|f_{*}^{n}(F)\right\| \leqq 8^{-n} \sqrt{ } 130$, it follows that $X$ is smooth in $B(8)-\{0\}$ and continuous in $B(8)$. The smoothness of $X$ implies that each point in $B(8)-\{0\}$ has a unique integral curve of $X$ passing through it. In view of (4a) none of these integral curves can reach the origin. For if $x \in(B(1)-\{0\})$, then for some $n>0, x \in\left(f^{n}(T)-f^{n+1}(T)\right)$ which is an invariant set bounded away from the origin. Consequently, $X$ has a unique integral curve through each point in a neighborhood of the origin and so generates a local flow. Since each nonstationary orbit of this flow is contained in an invariant manifold which is bounded away from $0, X$ generates an $\mathrm{NN}$-flow about 0 . This completes the proof.

\section{REFERENCES}

1. P. Hartman, Ordinary differential equations, Wiley, New York, 1964. MR 30 \#1270.

2. J. Milnor, Topology from the differentiable viewpoint, Univ. of Virginia Press, Charlottesville, Va., 1965.

3. J. R. Munkres, Elementary differential topology, Ann. of Math. Studies, no. 54, Princeton Univ. Press, Princeton, N.J., 1963. MR 29 \#623.

4. S. Sternberg, Lectures on differential geometry, Prentice-Hall, Englewood Cliffs, N.J., 1964. MR 33 \#1797.

Department of Mathematics, Newark College of Engineering, Newark, NEW JERSEY 07102 